\title{
THE CLASSICAL LIMIT OF THE HEISENBERG AND TIME-DEPENDENT HARTREE-FOCK EQUATIONS: THE WICK SYMBOL OF THE SOLUTION
}

\author{
Laurent Amour, Mohamed Khodja and Jean Nourrigat
}

\begin{abstract}
This paper is concerned with the Wick symbol of time evolving quantum observables. The time dynamics is following either the Heisenberg equation relative to the Schrödinger Hamiltonian, or the time-dependent Hartree-Fock equation. Under very weak assumptions, we prove that the Wick symbol approximatively follows the classical mechanics laws when the semiclassical parameter $h$ tends to zero. For the Heisenberg equation, this is a form of what is commonly called the Ehrenfest theorem. These statements have to be understood in a weaker sense than usual and in return, we do not assume that the Weyl symbol of the initial observable belongs to a class allowing the use of the Egorov theorem.
\end{abstract}

\section{Introduction}

The aim of this paper is twofold: to prove, under minimal hypotheses, a version of Ehrenfest theorem, and to give a similar result for the time-dependent HartreeFock equation (TDHF). We first consider a quantum observable (bounded operator) $A_{h}(t),(t \in \mathbb{R}, h>0)$, evolving according to the Heisenberg equation associated to the Schrödinger Hamiltonian (see (1.2)). Generally speaking, the Ehrenfest theorem suggests that the average of this observable $A_{h}(t)$, taken on an $h$-dependent coherent state, approximatively follows, for small $h>0$, the classical mechanics laws [7]. The average of an observable on coherent states is related to its Wick symbol. The first goal of this work is to obtain minimal hypotheses on the initial data $A_{h}(0)$, in order that the idea conveyed by Ehrenfest is asymptotically verified when $h$ tends to 0 .

A precise statement of Ehrenfest theorem, using Egorov theorem for the proof, is given in $[4,13]$ (see also the bibliography therein). It is also noted ([4] and references therein) that the approximation by the classical mechanics laws is not valid uniformly for all time, but up to a maximal time called the Ehrenfest time. These results are obtained assuming that the family of observables $\left(A_{h}(0)\right)$ is a semiclassical pseudodifferential (PDO) operator (see [13] or [17]). Our first goal is therefore to give another precise statement of Ehrenfest theorem, weaker than the one in [13], but in assuming weaker hypotheses on $A_{h}(0)$ (Theorem 1.1). In particular, we do not assume that $A_{h}(0)$ is a PDO operator. It is known that an operator in $L^{2}\left(\mathbb{R}^{n}\right)$ is a PDO one in the standard class of Calderón and Vaillancourt, when all its iterated commutators with position and momentum operators are bounded in $L^{2}\left(\mathbb{R}^{n}\right)$ (Beals characterization theorem [2]). In our work, the main assumption is that the single commutators of $A_{h}(0)$ with position and momentum operators are bounded, without any hypotheses on the iterated commutators. Then we prove that, in some sense, the

Received by the editors May 24, 2012. 
Wick symbol of $A_{h}(t)$ "approximately follows the classical mechanics laws", although, with our hypotheses, it does not necessarily have a limit when $h$ tends to zero. This is our first result.

Next, we consider the quantum dynamics associated to TDHF. In that case, the classical mechanics laws should be the Vlasov equations. Similarly to Theorem 1.1, the second purpose of this paper is to show that the Wick symbol of a solution $\rho_{h}(t)$ to the TDHF equation approximatively follows the Vlasov equation (Theorem 2.1). We assume that the operator $\rho_{h}(0)$ is trace class, self-adjoint, nonnegative, with a trace equal to 1 . It is also assumed that the single commutators of $\rho_{h}(0)$ with the position and momentum operators are trace class. Then $\rho_{h}(t)$, solution to TDHF equation, is trace class for all $t$, and therefore its Wick symbol is in $L^{1}\left(\mathbb{R}^{2 n}\right)$, as well as all its derivatives. Then, we can prove that, in some sense, this Wick symbol approximately follows the Vlasov equation. This Theorem 2.1 is similar to Theorem 1.1 , with trace class operators instead of bounded ones.

Moreover, the second result (Theorem 2.1) is a continuation of our work in [1], where the Weyl symbol was considered instead of the Wick symbol. The work in [1] is devoted to the the Weyl symbol of a solution $\rho_{h}(t)$ to the TDHF equation, under the hypothesis that $\rho_{h}(0)$ is a PDO trace class operator belonging to the class studied by Rondeaux [14]. The motivation of the study of the Wick symbol rather than the Weyl symbol is the following. The space $L^{1}\left(\mathbb{R}^{2 n}\right)$ is the natural function space for kinetic equations such as the Vlasov equation. However, the Weyl symbol of a trace class operator is not necessarily in $L^{1}\left(\mathbb{R}^{2 n}\right)$, without the strong hypotheses made in [1]. To the contrary, the Wick symbol of a trace class operator always belongs to $L^{1}\left(\mathbb{R}^{2 n}\right)$, and the use of the Wick symbol allows us to take very weak hypotheses.

One of the tools used to prove both of these two results is the approximation of a bounded operator, verifying rather weak hypotheses, by PDO operators belonging to the class of Calderón and Vaillancourt [6]. It is a kind of convolution where translations are replaced by an action of the Heisenberg group. We have similar results with bounded operators replaced by trace class ones, and the class of [6] replaced by that of [14]. The details are found in Section 5.

The paper is organized as follows. The two main results are stated in Sections 1 and 2. In Section 3, we give an example of trace class operator whose Weyl symbol is not in $L^{1}\left(\mathbb{R}^{2 n}\right)$. The regularity properties of the Wick symbol of bounded or trace class operators are derived in Section 4. Section 5 is concerned with PDO approximation. Finally, Theorems 1.1 and 2.1 are proved in Sections 6 and 7, respectively.

\section{Statement of the first result: the case of bounded operators and Ehrenfest theorem}

In this section, the time evolution of the system is associated to the following $h$ dependent Hamiltonian $(h>0)$,

$$
\widehat{H}_{h}=-h^{2} \Delta+V
$$

where $V$ is a $C^{\infty}$ real-valued function on $\mathbb{R}^{n}$, which is bounded together with all of its derivatives. We also denote by $\widehat{H}_{h}$ the unique self-adjoint extension of this operator. Let $\left(A_{h}\right)_{h>0}$ be a family of bounded self-adjoint operators in $L^{2}\left(\mathbb{R}^{n}\right)$. The operator 
$A_{h}(t)$ corresponding to the evolution of the operator $A_{h}$ at the time $t$ is,

$$
A_{h}(t)=e^{i \frac{t}{h} \widehat{H}_{h}} A_{h} e^{-i \frac{t}{h} \widehat{H}_{h}} .
$$

Let us recall the standard statements of Ehrenfest theorem (see [7]). The coherent state centered at the point $X=(x, \xi)$ in $\mathbb{R}^{2 n}$, is the following function, depending on $h>0$,

$$
\Psi_{X, h}(u)=(\pi h)^{-n / 4} e^{-\frac{|u-x|^{2}}{2 h}} e^{\frac{i}{h} u . \xi-\frac{i}{2 h} x \cdot \xi}, \quad X=(x, \xi) \in \mathbb{R}^{2 n}, \quad u \in \mathbb{R}^{n} .
$$

The average taken on $\Psi_{X, h}$ of a bounded operator $A$ in $\mathcal{H}=L^{2}\left(\mathbb{R}^{n}\right)$ is,

$$
\sigma_{h}^{\text {wick }}(A)(X)=\left\langle A \Psi_{X, h}, \Psi_{X, h}\right\rangle .
$$

The function $\sigma_{h}^{\text {wick }}(A)$ is called the Wick symbol of $A$ (see [12]). The Wick calculus has been also used, for instance, in $[3,9,8,10,15,16]$.

According to the statement of Ehrenfest theorems, as one can see in [7], the average of $A_{h}(t)$ taken on coherent states is supposed to "approximatively follow the classical mechanics laws, as $h$ tends to 0".

Let us precise in which sense does this function follow the classical mechanics equations, under the limit $h$ tends to 0 . Let $\varphi_{t}(x, \xi)=\left(q_{t}(x, \xi), p_{t}(x, \xi)\right)$ denotes the Hamiltonian flow of the function $H(x, \xi)=|\xi|^{2}+V(x)$ starting at $(x, \xi)$, i.e., the solution to,

$$
q_{t}^{\prime}(x, \xi)=2 p(t, x, \xi), \quad p_{t}^{\prime}(x, \xi)=-(\nabla V)(q(t, x, \xi)),
$$

such that $q_{0}(x, \xi)=x, p_{0}(x, \xi)=\xi$. Under our hypotheses on $V$, the flow is globally defined. One can say that a function $f(x, \xi, t)$ "follows the classical mechanics laws", when $f(X, t)=f\left(\varphi_{t}(X), 0\right)$, for all $X \in \mathbb{R}^{2 n}$ and all $t \in \mathbb{R}$.

Consequently, to say that the Wick symbol of the observable $A_{h}(t)$ asymptotically follows the classical mechanics laws can be expressed by the fact that for every $t \in \mathbb{R}$ :

$$
\lim _{h \rightarrow 0}\left(\sigma_{h}^{\text {wick }}\left(A_{h}(t)\right)(X)-\sigma_{h}^{\text {wick }}\left(A_{h}(0)\right)\left(\varphi_{t}(X)\right)\right)=0 .
$$

Our goal is to prove (1.6) under the weakest possible hypotheses on the initial data $A_{h}(0)$.

This limit (1.6) is standard when the initial data $A_{h}(0)$ is a semiclassical PDO operator (see, for instance, [13] or [17]). We briefly recall this notion. Let us denote by $W^{m, p}$ the Sobolev space of functions that are in $L^{p}\left(\mathbb{R}^{n}\right)$ together with all derivatives of order less or equal to $m$ (in the sense of distributions). A semiclassical PDO operator is a family of operators formally defined by

$$
\left(A_{h} f\right)(x)=(2 \pi h)^{-n} \int_{\mathbb{R}^{2 n}} F_{h}\left(\frac{x+y}{2}, \xi\right) e^{\frac{i}{h}(x-y) \cdot \xi} f(y) d y d \xi, \quad x \in \mathbb{R}^{n},
$$

where $F_{h}$ is a family of functions belonging to the space $W^{\infty, \infty}\left(\mathbb{R}^{2 n}\right)$ and bounded in this same space independently of $h \in(0,1]$. According to Calderón and Vaillancourt [6], $A_{h}$ is well defined as a bounded operator in $\mathcal{H}$. In this paper, the relationship between the function $F_{h}$ (called symbol) and the operator $A_{h}$ defined by (1.7) will be written in the two equivalent following ways:

$$
A_{h}=O p_{h}^{\text {weyl }}\left(F_{h}\right), \quad F_{h}=\sigma_{h}^{\text {weyl }}\left(A_{h}\right)
$$


(The Weyl symbol of an arbitrary bounded operator in $\mathcal{H}$ is, a priori, a tempered distribution on $\mathbb{R}^{2 n}$.)

The standard Egorov theorem claims that:

$$
\lim _{h \rightarrow 0}\left(\sigma_{h}^{\text {weyl }}\left(e^{i \frac{t}{h} \widehat{H}_{h}} A_{h} e^{-i \frac{t}{h} \widehat{H}_{h}}\right)(X)-F_{h}\left(\varphi_{t}(X)\right)\right)=0,
$$

where $A_{h}=O p_{h}^{\text {weyl }}\left(F_{h}\right)$ is a semiclassical PDO operator, with $F_{h}$ bounded in $W^{\infty, \infty}\left(\mathbb{R}^{2 n}\right)$ independently of $h \in(0,1]$, where $\widehat{H}_{h}$ is defined in (1.1), and the flow $\varphi_{t}$ in (1.5).

The earliest version of the Egorov theorem says that, if $A$ is a PDO operator and $U$ an invertible Fourier Integral Operator, then $U^{-1} A U$ is a $\mathrm{PDO}$ operator (see Hörmander [11], volume IV, chapter 25). For a product like in (1.2), it can be proved, without the use of Fourier integral operators, that, if $A_{h}$ is a semiclassical PDO operator with a symbol $F_{h}$ bounded in $W^{\infty, \infty}\left(\mathbb{R}^{2 n}\right)$, then the right-hand side of $(1.2)$ enjoys the same property. The proof was given by Robert [13] and by Zworski [17].

In that case, the limit (1.6) follows from (1.9) since the Wick symbol is obtained from the Weyl symbol through a mollification:

$$
\sigma_{h}^{\text {wick }}(A)=e^{\frac{h}{4} \Delta} \sigma_{h}^{\text {weyl }}(A),
$$

where $\Delta$ is the Laplacian on $\mathbb{R}^{2 n}$. See Proposition 4.4 below, or $[4,13]$ for more details.

Let us explain now how to prove (1.6) with weaker hypotheses than above. The assumption $A=O p_{h}^{\text {weyl }}(F)$ with $F$ in $W^{\infty, \infty}\left(\mathbb{R}^{2 n}\right)$ is rather strong, and may be expressed in terms of commutators, in a standard way. Let $P_{j}(h)$ and $Q_{j}(h)$ be the momentum and the position operators,

$$
P_{j}(h)=\frac{h}{i} \frac{\partial}{\partial x_{j}}, \quad Q_{j}(h)=x_{j} .
$$

According to Beals characterization result [2], the fact that $A_{h}$ may be written as, $A_{h}=O p_{h}^{\text {weyl }}(F)$ with $F$ in $W^{\infty, \infty}\left(\mathbb{R}^{2 n}\right)$ is equivalent to the fact that the commutators $(\text { ad } P(h))^{\alpha}(\text { ad } Q(h))^{\beta} A$ are bounded (for all multi-indexes $\alpha, \beta$ ). Saying that $F$ (which may depend on $h$ ) stays bounded in $W^{\infty, \infty}\left(\mathbb{R}^{2 n}\right)$ is equivalent to the fact,

$$
h^{-(|\alpha|+|\beta|)}\left\|(\operatorname{ad} P(h))^{\alpha}(\operatorname{ad} Q(h))^{\beta} A_{h}\right\|_{\mathcal{L}(\mathcal{H})} \leq M_{\alpha \beta},
$$

with $M_{\alpha \beta}$ independent on $h$.

We shall prove the limit (1.6) under much weaker hypotheses than in the case where $A_{h}(0)$ is a semiclassical operator. Namely, only single commutators (instead of iterated commutators) of the operator $A_{h}$ with the operators $P_{j}(h)$ and $Q_{j}(h)$ defined in (1.10) are assumed to be bounded operators. Our estimates shall involve the following expression,

$$
I_{h}^{\infty}(A)=\frac{1}{h} \sum_{j=1}^{n}\left\|\left[P_{j}(h), A\right]\right\|_{\mathcal{L}(\mathcal{H})}+\left\|\left[Q_{j}(h), A\right]\right\|_{\mathcal{L}(\mathcal{H})} .
$$

The theorem below provides the key inequality needed to show (1.6) under weakened hypotheses. When $A$ is a bounded operator, we shall notice (see Proposition 4.1) that the function $\sigma_{h}^{\text {wick }}(A)$ is $C^{\infty}$ on $\mathbb{R}^{2 n}$ and we shall give precise estimates on its derivatives in terms of the parameter $h$ and of the norm of $A$. 
Theorem 1.1. For all operators $A$ in $\mathcal{L}(\mathcal{H})$, such that the commutators $\left[P_{j}(h), A\right]$ and $\left[Q_{j}(h), A\right]$ are bounded operators in $\mathcal{H}(1 \leq j \leq n)$, the operator $A_{h}(t)$ defined in (1.2) and the Hamiltonian flow $\varphi_{t}$ defined in (1.5) satisfy,

$$
\left\|\sigma_{h}^{\text {wick }}\left(A_{h}(t)\right)-\left(\sigma_{h}^{\text {wick }}(A)\right) \circ \varphi_{t}\right\|_{L^{\infty}\left(\mathbb{R}^{2 n}\right)} \leq C(t) \sqrt{h} I_{h}^{\infty}(A),
$$

where $t \mapsto C(t)$ is a function defined on $\mathbb{R}$, bounded over any compact set, depending only on $n$ and on $V$, and where $I_{h}^{\infty}(A)$ is defined in (1.11).

In order that (1.12) implies (1.6), it suffices to replace $A$ by a family of operators $A_{h}$, such that the right-hand side of (1.12) tends to 0 as $h$ goes to 0 . This assumption is satisfied when $A_{h}=O p_{h}^{\text {weyl }}\left(F_{h}\right)$ with $F_{h}$ in $W^{\infty, \infty}\left(\mathbb{R}^{2 n}\right)$, and uniformly bounded in $h \in(0,1]$.

\section{Statement of the second result: the case of trace class operators and the TDHF equation}

Before introducing the TDHF, let us first specify our solutions space. We shall denote by $\mathcal{L}^{1}(\mathcal{H})$ the space of all trace class operators on $\mathcal{H}=L^{2}\left(\mathbb{R}^{n}\right)$ and by $\mathcal{D}$ the subspace of operators $A$ in $\mathcal{L}^{1}(\mathcal{H})$, such that the commutator $[\Delta, A]$ is also in $\mathcal{L}^{1}(\mathcal{H})$, where $\Delta$ is the Laplacian operator.

We consider two real-valued functions $V$ and $W$ in $W^{\infty, \infty}\left(\mathbb{R}^{n}\right)$. For all $h>0$, we shall say that a function $t \mapsto \rho_{h}(t)$ being $C^{1}$ from $\mathbb{R}$ into $\mathcal{L}^{1}(\mathcal{H})$ is a classical solution to the TDHF (according to the terminology of Bove-da Prato-Fano [5]) when this mapping is also continuous on $\mathbb{R}$ into $\mathcal{D}$ and if

$$
i h \frac{\partial}{\partial t} \rho_{h}(t)=-h^{2}\left[\Delta, \rho_{h}(t)\right]+\left[V_{q}\left(\rho_{h}(t)\right), \rho_{h}(t)\right]
$$

where $V_{q}\left(\rho_{h}(t)\right)$ denotes the multiplication operator by the function

$$
V_{q}\left(x, \rho_{h}(t)\right)=V(x)+\operatorname{Tr}\left(W_{x} \rho_{h}(t)\right)
$$

where $W_{x}$ is the multiplication operator by the function $y \mapsto W(x-y)$.

We consider a family $\left(\rho_{h}(t)\right)_{h>0}$ of classical solutions to (TDHF). We suppose that the operator $\rho_{h}(0)$ is trace class, self-adjoint $\geq 0$ with a trace equal to 1 . We set:

$$
u_{h}(X, t)=(2 \pi h)^{-n} \sigma_{h}^{\text {wick }}\left(\rho_{h}(t)\right)(X) .
$$

As we shall see in Section 4, this function lies in $W^{\infty, 1}\left(\mathbb{R}^{2 n}\right)$, it is nonnegative and

$$
\int_{\mathbb{R}^{2 n}} u_{h}(X, t) d X=1 .
$$

We denote by $v_{h}(X, t)$ the solution to the Cauchy problem for the Vlasov equation:

$$
\begin{gathered}
\frac{\partial v_{h}}{\partial t}+2 \sum_{j=1}^{n} \xi_{j} \frac{\partial v_{h}}{\partial x_{j}}-\sum_{j=1}^{n} \frac{\partial V_{c l}\left(x, v_{h}(., t)\right)}{\partial x_{j}} \frac{\partial v_{h}}{\partial \xi_{j}}=0 \\
v_{h}(X, 0)=u_{h}(X, 0) .
\end{gathered}
$$

We have set:

$$
V_{c l}\left(x, v_{h}(., t)\right)=V(x)+\int_{\mathbb{R}^{2 n}} W(x-y) v_{h}(y, \eta, t) d y d \eta .
$$

The function $v_{h}(., t)$ is itself in $L^{1}\left(\mathbb{R}^{2 n}\right)$, it is nonnegative and its integral equals to 1 . 
The counterpart of Ehrenfest theorem for a family of solutions to (TDHF) consists into saying that the function $u_{h}(., t)$ defined in $(2.3)$, satisfies approximately the Vlasov equation when $h$ tends to 0 . This point of view may be specified in different ways. Our aim is to compare, as in Theorem 1.1, the functions $u_{h}(., t)$ and $v_{h}(., t)$ and to show that, under suitable assumptions on $\rho_{h}(0)$, their difference in norm tends to 0 . Since we are concerned with trace class operators it makes sense that the norm involve to estimate the difference $u_{h}(., t)-v_{h}(., t)$ is the $L^{1}\left(\mathbb{R}^{2 n}\right)$ norm. A first answer to this issue is given in [1] where we assume that the operator $\rho_{h}(0)$ is a PDO operator belonging to the Rondeaux class [14] (with a modification to take into account the parameter $h$ ). The article [1] also gives an asymptotic expansion at any order of $u_{h}(., t)-v_{h}(., t)$. We consider here this problem with a weaker hypothesis than the one in [1]. We are only assuming that the commutators $\left[P_{j}(h), \rho_{h}(0)\right]$ and $\left[Q_{j}(h), \rho_{h}(0)\right]$ are trace class operators. All these estimates shall use the expression,

$$
I_{h}^{t r}\left(\rho_{h}(0)\right)=\frac{1}{h} \sum_{j=1}^{n}\left\|\left[P_{j}(h), \rho_{h}(0)\right]\right\|_{\mathcal{L}^{1}(\mathcal{H})}+\left\|\left[Q_{j}(h), \rho_{h}(0)\right]\right\|_{\mathcal{L}^{1}(\mathcal{H})} .
$$

We are now ready to state the main theorem of this section.

Theorem 2.1. Let $\left(\rho_{h}(t)\right)_{h>0}$ be a family of classical solutions to (TDHF) with realvalued potentials $V$ and $W$ in $W^{\infty, \infty}\left(\mathbb{R}^{2 n}\right)$. We suppose that the operator $\rho_{h}(0)$ is trace class, self-adjoint $\geq 0$, with a trace equal to 1 . We assume that all the commutators $\left[P_{j}(h), \rho_{h}(0)\right]$ and $\left[Q_{j}(h), \rho_{h}(0)\right]$ are trace class operators. Then, there exists a function $t \mapsto C(t)$, bounded on any compact set of $\mathbb{R}$ such that, for all $h \in(0,1]$ and for all $t \in \mathbb{R}$,

$$
\left\|u_{h}(., t)-v_{h}(., t)\right\|_{L^{1}\left(\mathbb{R}^{2 n}\right)} \leq C(t) \sqrt{h} I_{h}^{t r}\left(\rho_{h}(0)\right) e^{C(t) I_{h}^{t r}\left(\rho_{h}(0)\right)} .
$$

An immediate consequence is the next corollary.

Corollary 2.2. Under the assumptions of Theorem 2.1, if $I_{h}^{t r}\left(\rho_{h}(0)\right)$ remains bounded when $h$ tends to 0 , then we have,

$$
\lim _{h \rightarrow 0}\left\|u_{h}(., t)-v_{h}(., t)\right\|_{L^{1}\left(\mathbb{R}^{2 n}\right)}=0,
$$

for all $t \in \mathbb{R}$.

If $\rho_{h}(0)$ is a PDO operator of the form $\rho_{h}(0)=(2 \pi h)^{n} O p_{h}^{\text {weyl }}\left(F_{h}\right)$, with $F_{h}$ in $W^{\infty, 1}\left(\mathbb{R}^{2 n}\right)$ and uniformly bounded in $h$, then the assumption in corollary 2.2 is always satisfied. This is a consequence of the analogous result of the Beals characterization given in [14]. The dependence on the parameter $h$ is considered in [1].

Theorem 2.1 shall be proved in Section 7 .

\section{A counterexample}

Expanding on an idea of Rondeaux [14], we shall give an example of a trace class operator having a Weyl symbol not being in $L^{1}\left(\mathbb{R}^{2 n}\right)$. We first point out some properties of the Weyl symbol of a trace class operator. These properties are essential in the choice of the counterexample.

Proposition 3.1. If $A$ is trace class, then its Weyl symbol is a continuous function on $\mathbb{R}^{2 n}$ going to 0 at infinity and also belonging to $L^{2}\left(\mathbb{R}^{2 n}\right)$. If this function is also nonnegative then it is necessarily in $L^{1}\left(\mathbb{R}^{2 n}\right)$. 
Proof. If $A$ is trace class then it is also Hilbert-Schmidt and it is well-known that its Weyl symbol belongs to $L^{2}\left(\mathbb{R}^{2 n}\right)$ (cf. Robert [13]). This symbol is bounded since, when $A$ is trace class then we have

$$
\sigma_{h}^{\text {weyl }}(A)(X)=2^{n} \operatorname{Tr}\left(A \Sigma_{X h}\right), \quad X \in \mathbb{R}^{2 n},
$$

where $\Sigma_{X}$ is the operator in $\mathcal{H}$ defined in (4.15). The fact that it is continuous and that it is going to 0 at infinity is easily verified using (3.1), for an operator written as $u \mapsto A(u)=\langle u, \varphi\rangle \psi$, where $\varphi$ and $\psi$ are in $\mathcal{S}\left(\mathbb{R}^{n}\right)$. One then concludes this point by density, first for any finite rank $A$, then for any trace class operator since the set of finite rank operators is dense in $\mathcal{L}^{1}(\mathcal{H})$. For all functions $F$ in $W^{\infty, 1}\left(\mathbb{R}^{2 n}\right)$ and $G$ in $W^{\infty, \infty}\left(\mathbb{R}^{2 n}\right)$, it is well-known (cf. Robert [13]) that

$$
\operatorname{Tr}\left(O p_{h}^{\text {weyl }}(F) \circ O p_{h}^{\text {weyl }}(G)\right)=(2 \pi h)^{-n} \int_{\mathbb{R}^{2 n}} F(X) G(X) d X .
$$

Let $A$ be a trace class operator and $G$ be a $C^{\infty}$ function on $\mathbb{R}^{2 n}$ with compact support. From Theorem 5.2, there exists a sequence of functions $\left(F_{j}\right)$ in $W^{\infty, 1}\left(\mathbb{R}^{2 n}\right)$, such that the sequence of operators $O p_{h}^{\text {weyl }}\left(F_{j}\right)$ converges to $A$ in $\mathcal{L}^{1}(\mathcal{H})$, implying from (3.1) that $F_{j}$ converges uniformly to $\sigma_{h}^{\text {weyl }}(A)$. It is then deduced that

$$
\operatorname{Tr}\left(A \circ O p_{h}^{\text {weyl }}(G)\right)=(2 \pi h)^{-n} \int_{\mathbb{R}^{2 n}} \sigma_{h}^{\text {weyl }}(A)(X) G(X) d X .
$$

We replace $G$ by an increasing sequence $G_{N}$ of $C^{\infty}$ functions on $\mathbb{R}^{2 n}$, with compact support and converging pointwise to 1 when $N$ goes to $+\infty$, the functions $\left|\partial_{x}^{\alpha} \partial_{\xi}^{\beta} G_{N}\right|$ being all uniformly bounded on $N$. When $\sigma_{h}^{\text {weyl }}(A) \geq 0$, we deduce from (3.3) that

$$
0 \leq(2 \pi h)^{-n} \int_{\mathbb{R}^{2 n}} \sigma_{h}^{\text {weyl }}(A)(X) G_{N}(X) d X \leq\|A\|_{\mathcal{L}^{1}(\mathcal{H})}\left\|O p_{h}^{\text {weyl }}\left(G_{N}\right)\right\|_{\mathcal{L}(\mathcal{H})} .
$$

According to Calderón and Vaillancourt [6], the right-hand side remains bounded as $N$ tends $+\infty$. If the function $\sigma_{h}^{\text {weyl }}(A)$ is nonnegative then it is in $L^{1}\left(\mathbb{R}^{2 n}\right)$.

Proposition 3.1 and the results of Rondeaux [14] suggest the construction of an example of a trace class operator having a Weyl symbol not being in $L^{1}\left(\mathbb{R}^{2 n}\right)$. We may assume that $h=1$. Let $\alpha$ be a real number such that $\frac{n}{2}<\alpha \leq n$. We define the operator $P$ by

$$
P=O p_{1}^{\text {weyl }}(p), \quad p(x, \xi)=\frac{e^{2 i x . \xi}}{\left(1+|x|^{2}+|\xi|^{2}\right)^{\alpha}}, \quad(x, \xi) \in \mathbb{R}^{2 n} .
$$

The function $p$ is not in $L^{1}\left(\mathbb{R}^{2 n}\right)$. For all $\lambda>0$, we define an operator $A_{\lambda}$ by,

$$
A_{\lambda}=O p_{1}^{\text {weyl }}\left(a_{\lambda}\right), \quad a_{\lambda}(x, \xi)=e^{2 i x \xi-\lambda\left(|x|^{2}+|\xi|^{2}\right)} .
$$

We have

$$
P=\frac{1}{\Gamma(\alpha)} \int_{0}^{\infty} e^{-\lambda} \lambda^{\alpha-1} A_{\lambda} d \lambda
$$

provided that we verify the convergence. From definition (1.7) the integral kernel of $A_{\lambda}$ is the function $K_{\lambda}$ defined by

$$
K_{\lambda}(x, y)=(2 \pi)^{-n} \int_{\mathbb{R}^{n}} e^{i(x-y) \cdot \xi} a_{\lambda}\left(\frac{x+y}{2}, \xi\right) d \xi .
$$


An explicit computation shows that

$$
K_{\lambda}(x, y)=(2 \pi \lambda)^{-n / 2} e^{-\left(a(\lambda)|x|^{2}+b(\lambda)|y|^{2}+2 c(\lambda) x . y\right)}, a(\lambda)=\frac{\lambda}{4}+\frac{1}{\lambda}, b(\lambda)=c(\lambda)=\frac{\lambda}{4} .
$$

We can express the operator $A_{\lambda}$ as a product,

$$
A_{\lambda}=(2 \pi \lambda b(\lambda))^{-n / 2} T_{a(\lambda)} \circ B_{\lambda} \circ T_{b(\lambda)}^{-1} \circ S,
$$

where for all $a>0,\left(T_{a} f\right)(x)=f(x \sqrt{a}), S f(x)=f(-x)$ and

$$
\left(B_{\lambda} f\right)(x)=\int_{\mathbb{R}^{n}} e^{-\left(|x|^{2}+|y|^{2}\right)+2 \mu(\lambda) x \cdot y} f(y) d y, \quad \mu(\lambda)=\frac{c(\lambda)}{\sqrt{a(\lambda) b(\lambda)}} .
$$

The operator $B_{\lambda}$ is self-adjoint $\geq 0$ (since $0<\mu(\lambda)<1$ and $B(\lambda)$ may be identified, up to a multiplicative constant, to the exponential of an harmonic oscillator). We then see that

$$
\left\|B_{\lambda}\right\|_{\mathcal{L}^{1}(\mathcal{H})}=\operatorname{Tr}\left(B_{\lambda}\right)=\int_{\mathbb{R}^{n}} e^{-2(1-\mu(\lambda))|x|^{2}} d x=\left(\frac{\pi}{2(1-\mu(\lambda))}\right)^{n / 2} .
$$

From (3.6) we have

$$
\left\|A_{\lambda}\right\|_{\mathcal{L}^{1}(\mathcal{H})} \leq(2 \pi \lambda b(\lambda))^{-n / 2}\left\|T_{a(\lambda)}\right\|_{\mathcal{L}(\mathcal{H})}\left\|B_{\lambda}\right\|_{\mathcal{L}^{1}(\mathcal{H})}\left\|T_{b(\lambda)}^{-1}\right\|_{\mathcal{L}(\mathcal{H})}
$$

The right-hand side is polynomially increasing as $\lambda \rightarrow+\infty$ and it is $\mathcal{O}\left(\lambda^{-n / 2}\right)$ when $\lambda$ tends to 0 . With the choice of $\alpha>\frac{n}{2}$, the integral (3.5) converges in norm in $\mathcal{L}^{1}(\mathcal{H})$ and it properly defines a trace class operator $P$. The Weyl symbol of this operator is the function $p$ defined in (3.4) and does not belong to $L^{1}\left(\mathbb{R}^{2 n}\right)$.

\section{Differentiability of the Wick symbol}

For trace class operators, it is natural to give a variant of Ehrenfest theorem where the limit is understood in the $L^{1}\left(\mathbb{R}^{2 n}\right)$ sense. The above counterexample shows that the Weyl symbol is not suitable for this purpose without any additional hypothesis.

In this section, we shall show that the Wick symbol of a bounded operator (respectively of a trace class operator) in $L^{2}\left(\mathbb{R}^{n}\right)$ is a function in $W^{\infty, \infty}\left(\mathbb{R}^{2 n}\right)$ (resp. in $\left.W^{\infty 1}\left(\mathbb{R}^{2 n}\right)\right)$ and we shall give an upper bound for its derivatives.

Proposition 4.1. Let $A$ be a bounded operator in $\mathcal{H}$. Then the Wick symbol of $A$, namely, the function $\sigma_{h}^{\text {wick }}(A)$ defined on $\mathbb{R}^{2 n}$ by (1.4), is a $C^{\infty}$ function on $\mathbb{R}^{2 n}$, bounded together with all of its derivatives. For each multi-index $\alpha$ there exists $C_{\alpha}$ such that:

$$
\left\|\nabla^{\alpha} \sigma_{h}^{\text {wick }}(A)\right\|_{L^{\infty}\left(\mathbb{R}^{2 n}\right)} \leq C_{\alpha} h^{-|\alpha| / 2}\|A\|_{\mathcal{L}(\mathcal{H})} .
$$

Proof. We shall use the following function:

$$
S_{h}(A)(X, Y)=\frac{\left\langle A \Psi_{X, h}, \Psi_{Y, h}\right\rangle}{\left\langle\Psi_{X, h}, \Psi_{Y, h}\right\rangle},
$$

defined on $\mathbb{R}^{2 n} \times \mathbb{R}^{2 n}$, where the functions $\Psi_{X, h}$ are given in (1.3). If we identify $X=(x, \xi) \in \mathbb{R}^{n} \times \mathbb{R}^{n}$ with $x+i \xi \in \mathbf{C}^{n}$, we shall see that this function is holomorphic with respect to $X$, and antiholomorphic with respect to $Y$. The function $(X, Y) \rightarrow S_{h}(A)(X, \bar{Y})$, holomorphic with respect to both variables, is called Wick 
symbol in Folland [10]. The restriction of $S_{h}(A)$ to the diagonal is our $\sigma_{h}^{\text {wick }}(A)$. Direct computations shows that:

$$
\left\langle\Psi_{X, h}, \Psi_{Y, h}\right\rangle=e^{-\frac{1}{4 h}|X-Y|^{2}+\frac{i}{2 h} \operatorname{Im}(X . \bar{Y})} .
$$

In particular,

$$
\left|\left\langle\Psi_{X, h}, \Psi_{Y, h}\right\rangle\right|=e^{-\frac{1}{4 h}|X-Y|^{2}}, \quad\left\|\Psi_{X, h}\right\|=1
$$

Consequently,

$$
\left|S_{h}(A)(X, Y)\right|=e^{\frac{1}{4 h}|X-Y|^{2}}\left|\left\langle A \Psi_{X, h}, \Psi_{Y, h}\right\rangle\right| \leq e^{\frac{1}{4 h}|X-Y|^{2}}\|A\|_{\mathcal{L}(\mathcal{H})} .
$$

An important property verified by coherent states is that:

$$
\langle f, g\rangle=(2 \pi h)^{-n} \int_{\mathbb{R}^{2 n}}\left\langle f, \Psi_{X, h}\right\rangle\left\langle\Psi_{X, h}, g\right\rangle d X,
$$

for all $f$ and $g$ in $\mathcal{H}$. Applying this equality several times, we see that, for all $X$ and $Y$ :

$$
S_{h}(A)(X, Y)=(2 \pi h)^{-2 n} \int_{\mathbb{R}^{4 n}} \mathcal{B}_{h}(X, U, V, Y) S_{h}(A)(U, V) d U d V,
$$

where, for all $(X, U, V, Y)$ we have set:

$$
\mathcal{B}_{h}(X, U, V, Y)=\frac{\left\langle\Psi_{X, h}, \Psi_{U, h}\right\rangle\left\langle\Psi_{U, h}, \Psi_{V, h}\right\rangle\left\langle\Psi_{V, h}, \Psi_{Y, h}\right\rangle}{\left\langle\Psi_{X, h}, \Psi_{Y, h}\right\rangle} .
$$

Direct computations from (4.3) show that this function is holomorphic with respect to $X$ and antiholomorphic with respect to $Y$, and the same properties follow for $S_{h}(A)$. The function $\mathcal{B}_{h}$ plays the role of a modified Bergman kernel. From (4.3) we have:

$$
\mathcal{B}_{h}(X, U, V, X)=e^{-\frac{1}{2 h}(X-V)(\bar{X}-\bar{U})-\frac{1}{2 h}|U-V|^{2}} .
$$

We verify that:

$$
h^{m / 2}\left|\nabla_{X}^{\alpha} \mathcal{B}_{h}(X, U, V, X)\right| \leq C_{m} K_{m}(X, U, V, h) e^{-\frac{1}{4 h}|U-V|^{2}},
$$

where $m=|\alpha|$ and

$$
K_{m}(X, U, V, h)=\left(1+\frac{|X-U|+|X-V|}{\sqrt{h}}\right)^{m} e^{-\frac{1}{4 h}\left(|X-U|^{2}+|V-X|^{2}\right)} .
$$

Applying (4.7) restricted to the diagonal $X=Y$, it follows that:

$$
h^{m / 2}\left|\nabla_{X}^{\alpha} \sigma_{h}^{\text {wick }}(A)(X)\right| \leq C(2 \pi h)^{-2 n} \int_{\mathbb{R}^{4 n}}\left|\left\langle A \Psi_{U, h}, \Psi_{V, h}\right\rangle\right| K_{m}(X, U, V, h) d U d V .
$$

Bounding from above $\left|\left\langle A \Psi_{U, h}, \Psi_{V, h}\right\rangle\right|$ by the norm of $A$, we then obtain (4.1).

Proposition 4.2. If $A$ is in $\mathcal{L}^{1}(\mathcal{H})$, then its Wick symbol $\sigma_{h}^{\text {wick }}(A)$ belongs to $W^{\infty, 1}$ $\left(\mathbb{R}^{2 n}\right)$. For each multi-index $\alpha$, there exists $C_{\alpha}>0$ such that:

$$
(2 \pi h)^{-n}\left\|\nabla^{\alpha} \sigma_{h}^{\text {wick }}(A)\right\|_{L^{1}\left(\mathbb{R}^{2 n}\right)} \leq C_{\alpha} h^{-\frac{\alpha}{2}}\|A\|_{\mathcal{L}^{1}(\mathcal{H})} .
$$

Inequality (4.11) is proved when $\alpha=0$ by Rondeaux [14]. 
Proof. Similarly to Proposition 4.1, we have inequality (4.10). Integrating with respect to $X$ we obtain:

$$
\begin{aligned}
& h^{m / 2}(2 \pi h)^{-n}\left\|\nabla^{\alpha} \sigma_{h}^{\text {wick }}(A)\right\|_{L^{1}\left(\mathbb{R}^{2 n}\right)} \\
& \quad \leq C_{\alpha}(2 \pi h)^{-2 n} \int_{\mathbb{R}^{4 n}} \mid\left\langle A \Psi_{U, h}, \Psi_{V, h}\right\rangle G_{m}\left(\frac{U-V}{\sqrt{h}}\right) d U d V
\end{aligned}
$$

where

$$
G_{m}(U)=(1+|U|)^{m} e^{-\frac{|U|^{2}}{8}}
$$

where $m=|\alpha|$.

Since the function $G_{m}$ is in $L^{1}\left(\mathbb{R}^{2 n}\right)$, Proposition 4.2 is a consequence of the following lemma.

Lemma 4.3. Let $A$ be a trace class operator and $G$ be a function in $L^{1}\left(\mathbb{R}^{2 n}\right)$. Then we have:

$$
\begin{aligned}
(2 \pi h)^{-2 n} \int_{\mathbb{R}^{4 n}} \mid<A \Psi_{X, h}, \Psi_{Y, h} & >G\left(\frac{X-Y}{\sqrt{h}}\right) \mid d X d Y \\
& \leq(2 \pi)^{-n}\|G\|_{L^{1}\left(\mathbb{R}^{2 n}\right)}\|A\|_{\mathcal{L}^{1}(\mathcal{H})} .
\end{aligned}
$$

Proof. We can write $A=B_{1} B_{2}$ where $B_{1}$ and $B_{2}$ are two Hilbert-Schmidt operators. Using the fundamental identity (4.6) verified by the coherent states we see that, for all $X$ and $Y$ in $\mathbb{R}^{2 n}$,

$$
\left\langle A \Psi_{X, h}, \Psi_{Y, h}\right\rangle=\left\langle B_{2} \Psi_{X, h}, B_{1}^{\star} \Psi_{Y, h}\right\rangle=(2 \pi h)^{-n} \int_{\mathbb{R}^{2 n}} u_{Z h}(X) v_{Z h}(Y) d Z
$$

where we have set $u_{Z h}(X)=\left\langle B_{2} \Psi_{X, h}, \Psi_{Z, h}\right\rangle$ and $v_{Z h}(X)=\left\langle\Psi_{Z, h}, B_{1}^{\star} \Psi_{X, h}\right\rangle$. Let $I_{h}$ be the left-hand side of (4.12). From Schur lemma,

$$
I_{h} \leq(2 \pi h)^{-3 n} h^{n}\|G\|_{L^{1}\left(\mathbb{R}^{2 n}\right)} \int_{\mathbb{R}^{2 n}}\left\|u_{Z h}\right\|_{L^{2}\left(\mathbb{R}^{2 n}\right)}\left\|v_{Z h}\right\|_{L^{2}\left(\mathbb{R}^{2 n}\right)} d Z .
$$

From (4.6), we have $\left\|u_{Z h}\right\|_{L^{2}\left(\mathbb{R}^{2 n}\right)}=(2 \pi h)^{n / 2}\left\|B_{2}^{\star} \Psi_{Z, h}\right\|$ and $\left\|v_{Z h}\right\|_{L^{2}\left(\mathbb{R}^{2 n}\right)}=(2 \pi h)^{n / 2}$ $\left\|B_{1} \Psi_{Z, h}\right\|$. Consequently,

$$
I_{h} \leq(2 \pi h)^{-2 n} h^{n}\|G\|_{L^{1}\left(\mathbb{R}^{2 n}\right)} \int_{\mathbb{R}^{2 n}}\left\|B_{1} \Psi_{Z, h}\right\|\left\|B_{2}^{\star} \Psi_{Z, h}\right\| d Z .
$$

From the basic identity (4.6) for coherent states,

$$
\begin{aligned}
(2 \pi h)^{-n} \int_{\mathbb{R}^{2 n}}\left\|B_{j} \Psi_{Z, h}\right\|^{2} d Z & =(2 \pi h)^{-n} \int_{\mathbb{R}^{2 n}}\left\langle B_{j}^{\star} B_{j} \Psi_{Z, h}, \Psi_{Z, h}\right\rangle d Z \\
& =\operatorname{Tr}\left(B_{j}^{\star} B_{j}\right)=\left\|B_{j}\right\|_{\mathcal{L}^{2}(\mathcal{H})}^{2},
\end{aligned}
$$

where $\left\|B_{j}\right\|_{\mathcal{L}^{2}(\mathcal{H})}$ is the Hilbert-Schmidt norm of $B_{j}$. Therefore,

$$
I_{h} \leq(2 \pi)^{-n}\|G\|_{L^{1}\left(\mathbb{R}^{2 n}\right)}\left\|B_{1}\right\|_{\mathcal{L}^{2}(\mathcal{H})}\left\|B_{2}\right\|_{\mathcal{L}^{2}(\mathcal{H})} .
$$

Taking the infimum over all $A$ written as $A=B_{1} B_{2}$ we then obtain (4.12).

Let us now recall and give a short proof of the following proposition, even if it is standard. 
Proposition 4.4. The Wick symbol $\sigma_{h}^{\text {wick }}(A)$ of an operator $A$ is related to its Weyl symbol $\sigma_{h}^{\text {weyl }}(A)$ by:

$$
\sigma_{h}^{\text {wick }}(A)=e^{\frac{h}{4} \Delta} \sigma_{h}^{\text {weyl }}(A),
$$

where $\Delta$ is the Laplacian on $\mathbb{R}^{2 n}$.

Indeed, setting $F_{h}=\sigma_{h}^{\text {weyl }}(A)$, the expression in definition (1.7) for the Weyl calculus may be written as:

$$
A=(\pi h)^{-n} \int_{\mathbb{R}^{2 n}} F_{h}(Y) \Sigma_{Y h} d Y
$$

where, for all $Y=(y, \eta)$ in $\mathbb{R}^{2 n}, \Sigma_{Y h}$ is the symmetry operator defined by:

$$
\left(\Sigma_{Y h} f\right)(u)=e^{\frac{2 i}{h}(u-y) \xi} f(2 y-u), \quad Y=(y, \eta) \in \mathbb{R}^{2 n} .
$$

A direct computation shows that:

$$
\sigma_{h}^{\text {wick }}\left(\Sigma_{Y h}\right)(X)=<\Sigma_{Y h} \Psi_{X, h}, \Psi_{X, h}>=e^{-\frac{|X-Y|^{2}}{h}} .
$$

Equality (4.13) then follows.

\section{PDO approximation}

In this section, we shall show that the class of operators having a Weyl symbol in $W^{\infty, 1}\left(\mathbb{R}^{2 n}\right)$ (introduced by Rondeaux) is dense in the set of trace class operators, similarly to the fact that $W^{\infty, 1}\left(\mathbb{R}^{n}\right)$ is dense in $L^{1}\left(\mathbb{R}^{n}\right)$. The PDO approximation used for the proof is strongly connected with convolutions and may probably be also applied to Schatten classes. This technique is often employed in representation theory. However, as a function in $L^{\infty}\left(\mathbb{R}^{n}\right)$ needs to be continuous in order to be in the closure of $W^{\infty, \infty}\left(\mathbb{R}^{n}\right)$, a bounded operator needs additional hypotheses in order to be in the closure of the class of Calderón-Vaillancourt operators.

For all $X=(x, \xi)$ in $\mathbb{R}^{2 n}$ and for all $h>0$ let $W_{x, \xi, h}$ be the operator defined by:

$$
\left(W_{X, h} f\right)(u)=\left(W_{x, \xi, h} f\right)(u)=f(u-x) e^{\frac{i}{h} u \cdot \xi-\frac{i}{2 h} x \cdot \xi},
$$

for all $f \in L^{2}\left(\mathbb{R}^{n}\right)$. It is a standard representation of the Heisenberg group. Thus, the coherent state $\Psi_{X, h}$ verifies:

$$
\Psi_{X, h}=W_{X, h} \Psi_{0, h}
$$

We have, for all $X$ and $Y$ in $\mathbb{R}^{2 n}$,

$$
W_{X, h} W_{Y, h}=e^{\frac{i}{2 h} \sigma(X, Y)} W_{X+Y, h},
$$

where $\sigma$ is the symplectic form $\sigma((x, \xi),(y, \eta))=y . \xi-x . \eta$. For all operators $A$ in $\mathcal{L}(\mathcal{H})$ and for all $h>0$ let us define:

$$
\mathcal{T}_{h} A=(\pi h)^{-n} \int_{\mathbb{R}^{2 n}} e^{-\frac{|X|^{2}}{h}} W_{X, h} A W_{X, h}^{\star} d X
$$

We note that without further hypotheses $\left\langle\mathcal{T}_{h} A \phi, \psi\right\rangle$ makes sense for $\phi$ and $\psi$ in $\mathcal{S}\left(\mathbb{R}^{n}\right)$.

We begin with the case of bounded and trace class operators. Some mild hypotheses on their commutators with position and momentum operators are needed. 
Theorem 5.1. (a) We have:

$$
\left\|\mathcal{T}_{h} A\right\|_{\mathcal{L}(\mathcal{H})} \leq\|A\|_{\mathcal{L}(\mathcal{H})}
$$

for all operators $A$ in $\mathcal{L}(\mathcal{H})$ and for all $h>0$.

(b) When the commutators $\left[P_{j}(h), A\right]$ and $\left[Q_{j}(h), A\right]$ are bounded operators we have:

$$
\left\|A-\mathcal{T}_{h} A\right\|_{\mathcal{L}(\mathcal{H})} \leq \frac{C}{\sqrt{h}} \sum_{j=1}^{n}\left\|\left[P_{j}(h), A\right]\right\|_{\mathcal{L}(\mathcal{H})}+\left\|\left[Q_{j}(h), A\right]\right\|_{\mathcal{L}(\mathcal{H})} .
$$

(c) When the operator $A$ is trace class together with the commutators $\left[P_{j}(h), A\right]$ and $\left[Q_{j}(h), A\right]$, we have:

$$
\left\|A-\mathcal{T}_{h} A\right\|_{\mathcal{L}^{1}(\mathcal{H})} \leq \frac{C}{\sqrt{h}} \sum_{j=1}^{n}\left\|\left[P_{j}(h), A\right]\right\|_{\mathcal{L}^{1}(\mathcal{H})}+\left\|\left[Q_{j}(h), A\right]\right\|_{\mathcal{L}^{1}(\mathcal{H})} .
$$

(d) The Wick symbol of the operators $A$ and $\mathcal{T}_{h} A$ are related with:

$$
\sigma_{h}^{\text {wick }}\left(\mathcal{T}_{h} A\right)=e^{\frac{h}{4} \Delta} \sigma_{h}^{\text {wick }}(A) .
$$

The Weyl symbol of $\mathcal{T}_{h} A$ is equal to the Wick symbol of $A$.

Proof. Point (a) is clear since $W_{X h}$ is unitary. For any $\theta$ in $[0,1]$ define:

$$
T(\theta, h) A=(\pi h)^{-n} \int_{\mathbb{R}^{2 n}} e^{-\frac{|X|^{2}}{h}} W_{\theta X, h} A W_{\theta X, h}^{\star} d X .
$$

Thus, $T(1, h) A=T_{h} A$ and $T(0, h) A=A$. We verify that:

$$
\frac{\partial}{\partial \theta} W_{\theta X, h} A W_{\theta X, h}^{\star}=\frac{i}{h} \sum_{j=1}^{n}\left[x_{j} W_{\theta X, h}\left[P_{j}(h), A\right] W_{\theta X, h}^{\star}-\xi_{j} W_{\theta X, h}\left[Q_{j}(h), A\right] W_{\theta X, h}^{\star}\right] .
$$

Consequently,

$$
\begin{aligned}
\left\|A-\mathcal{T}_{h} A\right\|_{\mathcal{L}(\mathcal{H})} \leq & \frac{1}{h} \sum_{j=1}^{n}(\pi h)^{-n} \int_{\mathbb{R}^{2 n} \times[0,1]} e^{-\frac{|X|^{2}}{h}}\left[\left|x_{j}\right|\left\|\left[P_{j}(h), A\right]\right\|_{\mathcal{L}(\mathcal{H})}\right. \\
& \left.+\left|\xi_{j}\right|\left\|\left[Q_{j}(h), A\right]\right\|_{\mathcal{L}(\mathcal{H})}\right] d x d \xi d \theta \\
\leq & \frac{C}{\sqrt{h}} \sum_{j=1}^{n}\left\|\left(a d P_{j}(h)\right) A\right\|_{\mathcal{L}(\mathcal{H})}+\left\|\left(a d Q_{j}(h)\right) A\right\|_{\mathcal{L}(\mathcal{H})}
\end{aligned}
$$

proving point (b) and also point (c) with straightforward modifications. For the point (d) we see that:

$$
\begin{aligned}
\sigma_{h}^{\text {wick }}\left(\mathcal{T}_{h} A\right)(X) & =\left\langle\left(\mathcal{T}_{h} A\right) \Psi_{X, h}, \Psi_{X, h}\right\rangle=\left\langle\left(\mathcal{T}_{h} A\right) W_{X, h} \Psi_{0, h}, W_{X, h} \Psi_{0, h}\right\rangle \\
& =(\pi h)^{-n} \int_{\mathbb{R}^{2 n}} e^{-\frac{|Y|^{2}}{h}}\left\langle W_{Y, h} A W_{Y, h}^{\star} W_{X, h} \Psi_{0, h}, W_{X, h} \Psi_{0, h}\right\rangle d Y \\
& =(\pi h)^{-n} \int_{\mathbb{R}^{2 n}} e^{-\frac{|Y|^{2}}{h}}\left\langle A W_{X-Y, h} \Psi_{0, h}, W_{X-Y, h} \Psi_{0, h}\right\rangle d Y .
\end{aligned}
$$

We have used here (5.3). Consequently,

$$
\sigma_{h}^{\text {wick }}\left(\mathcal{T}_{h} A\right)(X)=(\pi h)^{-n} \int_{\mathbb{R}^{2 n}} e^{-\frac{|Y|^{2}}{h}} \sigma_{h}^{\text {wick }}(A)(X-Y) d Y,
$$


which is (5.8). According to Proposition 4.4 we also have:

$$
\sigma_{h}^{\text {wick }}\left(\mathcal{T}_{h} A\right)=e^{\frac{h}{4} \Delta} \sigma_{h}^{\text {weyl }}\left(\mathcal{T}_{h} A\right) .
$$

Since the operator $e^{\frac{h}{4} \Delta}$ is one to one, we deduce as it is mentioned that $\sigma_{h}^{\text {weyl }}\left(\mathcal{T}_{h} A\right)=$ $\sigma_{h}^{\text {wick }}(A)$.

Next, we consider the case of trace class operators without additional assumptions. The result below does not have any counterpart in the case of bounded operators.

Theorem 5.2. The space of operators written as $O P_{h}^{\text {weyl }}(F)$ with $F$ in $W^{\infty, 1}\left(\mathbb{R}^{2 n}\right)$ is dense in the space $\mathcal{L}^{1}(\mathcal{H})$ of trace class operators.

Proof. For this purpose, we modify the PDO approximation and we set:

$$
\mathcal{T}_{\lambda}^{\prime} A=(\pi \lambda)^{-n} \int_{\mathbb{R}^{2 n}} e^{-\frac{|X|^{2}}{\lambda}} W_{X, 1} A W_{X, 1}^{\star} d X
$$

for all $\lambda>0$ and for all trace class operators $A$. Let us show that:

$$
\lim _{\lambda \rightarrow 0}\left\|\mathcal{T}_{\lambda}^{\prime}(A)-A\right\|_{\mathcal{L}^{1}(\mathcal{H})}=0
$$

for all trace class operators $A$. Since we clearly have

$$
A=(\pi \lambda)^{-n} \int_{\mathbb{R}^{2 n}} e^{-\frac{|X|^{2}}{\lambda}} A d X
$$

then we see that,

$$
\begin{aligned}
& \left\|\mathcal{T}_{\lambda}^{\prime}(A)-A\right\|_{\mathcal{L}^{1}(\mathcal{H})} \leq(\pi \lambda)^{-n} \int_{|X|<\delta} e^{-\frac{|X|^{2}}{\lambda}}\left\|W_{X, h} A W_{X, h}^{\star}-A\right\|_{\mathcal{L}^{1}(\mathcal{H})} \cdots \\
& \quad+(\pi \lambda)^{-n} \int_{|X|>\delta} e^{-\frac{|X|^{2}}{\lambda}}\left[\left\|W_{X, \lambda} A W_{X, h}^{\star}\right\|_{\mathcal{L}^{1}(\mathcal{H})}+\|A\|_{\mathcal{L}^{1}(\mathcal{H})}\right] d X
\end{aligned}
$$

for all $\delta>0$ and for all $\lambda>0$. For all trace class operators $A$ and for all $\varepsilon>0$, there exists $\delta>0$ such that:

$$
|X|<\delta \Longrightarrow\left\|W_{X, 1} A W_{X, 1}^{\star}-A\right\|_{\mathcal{L}^{1}(\mathcal{H})}<\varepsilon .
$$

Indeed, this property is first verified when $A$ is of the form $f \rightarrow\langle f, \varphi\rangle \psi$ with $\varphi$ and $\psi$ in $\mathcal{S}\left(\mathbb{R}^{n}\right)$, it is next derived by density for finite-rank operators and then, by density again for trace class operators. Besides, $\delta>0$ being fixed, we have:

$$
\lim _{\lambda \rightarrow 0}(\pi \lambda)^{-n} \int_{|X|>\delta} e^{-\frac{|X|^{2}}{\lambda}}\left[\left\|W_{X, 1} A W_{X, 1}^{\star}\right\|_{\mathcal{L}^{1}(\mathcal{H})}+\|A\|_{\mathcal{L}^{1}(\mathcal{H})}\right] d X=0 .
$$

The limit in (5.9) is then easily obtained. From (5.3), for all $X$ in $\mathbb{R}^{2 n}$ we have:

$$
\begin{aligned}
W_{X, 1} \mathcal{T}_{\lambda}^{\prime}(A) W_{X, 1}^{\star} & =(\pi \lambda)^{-n} \int_{\mathbb{R}^{2 n}} e^{-\frac{|Y|^{2}}{\lambda}} W_{X+Y, 1} A W_{X+Y, 1}^{\star} d Y \\
& =(\pi \lambda)^{-n} \int_{\mathbb{R}^{2 n}} e^{-\frac{|X-Z|^{2}}{\lambda}} W_{Z, 1} A W_{Z, 1}^{\star} d Z .
\end{aligned}
$$


Consequently, for all $\lambda>0$, the mapping $X \rightarrow W_{X, 1} \mathcal{T}_{\lambda}^{\prime}(A) W_{X, 1}^{\star}$ is $C^{\infty}$ from $\mathbb{R}^{2 n}$ into $\mathcal{L}^{1}(\mathcal{H})$. We see, setting $X=(x, \xi)$ as the variable of $\mathbb{R}^{2 n}$ :

$$
\begin{gathered}
\frac{\partial}{\partial x_{j}} W_{X, 1} \mathcal{T}_{\lambda}^{\prime}(A) W_{X, 1}^{\star}=-W_{X, 1}\left[P_{j}(1), \mathcal{T}_{\lambda}^{\prime}(A)\right] W_{X, 1}^{\star} \\
\frac{\partial}{\partial \xi_{j}} W_{X, 1} \mathcal{T}_{\lambda}^{\prime}(A) W_{X, 1}^{\star}=W_{X, 1}\left[Q_{j}(1), \mathcal{T}_{\lambda}^{\prime}(A)\right] W_{X, 1}^{\star},
\end{gathered}
$$

where $P_{j}(1)$ is the operator of differentiation with respect to $u_{j}$ and $Q_{j}(1)$ is the multiplication operator by $u_{j}$. Consequently, all order iterated commutators $\mathcal{T}_{\lambda}^{\prime}(A)$ with the position and momentum operators $P_{j}(1)$ and $Q_{j}(1)$ are trace class. From the result of characterization of Rondeaux [14] (the analogue of Beals characterization for trace class operators) it follows that $\mathcal{T}_{\lambda}^{\prime}(A)$ is written as $O p_{1}^{\text {weyl }}\left(F_{\lambda}\right)$, with $F_{\lambda}$ in $W^{\infty, 1}\left(\mathbb{R}^{2 n}\right)$, for all $\lambda>0$.

\section{Proof of Theorem 1.1}

Let $A$ be an operator in $\mathcal{L}(\mathcal{H})$ satisfying the assumptions in Theorem 1.1. Set $F_{h}(X)=$ $\sigma_{h}^{\text {wick }}(A)(X)$. This function is $C^{\infty}$ from Proposition 4.1. Denoting by $\varphi_{t}$ the Hamiltonian flow associated to the function $H(x, \xi)=|\xi|^{2}+V(x)$, we shall use the following function and operators:

$$
w_{h}(X, t)=F_{h}\left(\varphi_{t}(X)\right), \quad C_{h}(t)=O p_{h}^{\text {weyl }}\left(w_{h}(., t)\right) .
$$

We shall also use the operator $\mathcal{T}_{h} A$ appearing in Section 5 and the following operator:

$$
B_{h}(t)=e^{i \frac{t}{h} \widehat{H}_{h}} \mathcal{T}_{h}(A) e^{-i \frac{t}{h} \widehat{H}_{h}} .
$$

We shall compare Wick symbols of the operators $A_{h}(t), B_{h}(t)$ and $C_{h}(t)$ and then compare the Wick symbol of $C(t, h)$ with the function $w_{h}(., t)$. This is the purpose of the three steps below.

First step. We have, from Theorem 5.1,

$$
\begin{aligned}
\left\|\sigma_{h}^{\text {wick }}\left(A_{h}(t)-B_{h}(t)\right)\right\|_{L^{\infty}\left(\mathbb{R}^{2 n}\right)} & \leq\left\|A_{h}(t)-B_{h}(t)\right\|_{\mathcal{L}(\mathcal{H})}=\left\|A-T_{h}(A)\right\|_{\mathcal{L}(\mathcal{H})} \cdots \\
& \leq C \sqrt{h} I_{h}^{\infty}(A),
\end{aligned}
$$

where $I_{h}^{\infty}(A)$ is defined in (1.11).

Second step. The comparison of $B_{h}(t)$ and $C_{h}(t)$ comes from Egorov theorem. Nevertheless, this requires some precisions due to unusual estimates satisfied by the derivatives of $w_{h}(., t)$ that we first need to specify. Since $w_{h}(., 0)=\sigma_{h}^{\text {wick }}(A)$ we deduce that:

$$
\frac{\partial w_{h}(., 0)}{\partial x_{j}}=\frac{i}{h} \sigma_{h}^{\text {wick }}\left(\left[P_{j}(h), A\right]\right), \quad \frac{\partial w_{h}(., 0)}{\partial \xi_{j}}=-\frac{i}{h} \sigma_{h}^{\text {wick }}\left(\left[Q_{j}(h), A\right]\right) .
$$

Applying Proposition 4.1 to the above commutators, we see when $k \geq 1$ that:

$$
\left\|\nabla^{k} w_{h}(., 0)\right\|_{L^{\infty}\left(\mathbb{R}^{2 n}\right)} \leq C_{\alpha \beta} h^{1-(k+1) / 2} I_{h}^{\infty}(A) .
$$

The derivatives of order greater than one of the Hamiltonian flow $\varphi_{t}$ associated to the symbol $H(x, \xi)=|\xi|^{2}+V(x)$ are bounded in $\mathbb{R}^{2 n}$ with a bound equal to $\mathcal{O}\left(1+t^{2}\right)$. 
For every multi-indexes $\alpha$, there exists $M_{\alpha}(t)$ such that:

$$
\left\|\nabla^{\alpha} w_{h}(., t)\right\|_{L^{\infty}\left(\mathbb{R}^{2 n}\right)} \leq M_{\alpha}(t) h^{1-(\alpha+1) / 2} I_{h}^{\infty}(A) .
$$

The operators $B_{h}(t)$ and $C_{h}(t)$ satisfy:

$$
\begin{gathered}
-i h \frac{\partial B_{h}(t)}{\partial t}=\left[\widehat{H}_{h}, B(t, h)\right] \\
-i h \frac{\partial C_{h}(t)}{\partial t}=-i h O p_{h}^{\text {weyl }}\left(\partial_{t} w_{h}(., t)\right)=-i h O p_{h}^{\text {weyl }}\left(\left\{H, w_{h}(., t)\right\}\right) .
\end{gathered}
$$

For all functions $F$ and $G$ in $W^{\infty, \infty}\left(\mathbb{R}^{2 n}\right)$, let $\widehat{R}_{h}^{(2)}(F, G)$ be the operator defined by:

$$
\left[O p_{h}^{\text {weyl }}(F), O p_{h}^{\text {weyl }}(G)\right]=\frac{h}{i} O p_{h}^{\text {weyl }}(\{F, G\})+\widehat{R}_{h}^{(2)}(F, G) .
$$

From standard results on the Weyl calculus, this operator satisfies the following estimate:

$$
\left\|\widehat{R}_{h}^{(2)}(F, G)\right\|_{\mathcal{L}(\mathcal{H})} \leq C \sum_{\substack{j \geq 3, k \geq 3 \\ 6 \leq j+k \leq 6 n+10}} h^{(j+k) / 2}\left\|\nabla^{j} F\right\|_{L^{\infty}\left(\mathbb{R}^{2 n}\right)}\left\|\nabla^{k} G\right\|_{L^{\infty}\left(\mathbb{R}^{2 n}\right)} .
$$

It suffices to carefully follow the steps of the proof in [13], or, for a detailed proof, the appendix $\mathrm{B}$ of $[1]$. With these notations, one may write:

$$
-i h \frac{\partial C_{h}(t)}{\partial t}-\left[\widehat{H}_{h}, C_{h}(t)\right]=\widehat{R}_{h}^{(2)}\left(H, w_{h}(., t)\right) .
$$

Note that $\widehat{R}_{h}^{(2)}\left(\Delta, w_{h}(., t)\right)=0$ and consequently:

$$
\widehat{R}_{h}^{(2)}\left(H, w_{h}(., t)\right)=\widehat{R}_{h}^{(2)}\left(V, w_{h}(., t)\right) .
$$

We then may apply inequality (6.7) with the functions $F=V$ and $G=w_{h}(., t)$. The inequality (6.7), those in (6.4) which are verified by $w_{h}(., t)$, and the fact that all derivatives of $V$ are bounded allows us to write:

$$
\left\|\widehat{R}_{h}^{(2)}\left(H, G_{h}(., t)\right)\right\|_{\mathcal{L}(\mathcal{H})} \leq M(t) h^{3 / 2} I_{h}^{\infty}(A) .
$$

From Theorem 5.1, the operator $\mathcal{T}_{h} A$ appearing in Section 5 has a Weyl symbol equal to the Wick symbol of $A$. Consequently, the Weyl symbol of $B_{h}(0)=\mathcal{T}_{h}(A)$ and the one of $C_{h}(0)$, which is $F_{h}=\sigma_{h}^{\text {wick }}(A)$ are equal. Thus:

$$
B_{h}(0)=C_{h}(0) \text {. }
$$

From (6.5), (6.8) and (6.10), Duhamel principle implies:

$$
i h\left[B_{h}(t)-C_{h}(t)\right]=\int_{0}^{t} e^{i \frac{t-s}{h} \widehat{H}_{h}} \widehat{R}_{h}^{(2)}\left(H, G_{h}(., t)\right) e^{-i \frac{t-s}{h} \widehat{H}_{h}} d s .
$$

Consequently, when $t>0$,

$$
\left\|B_{h}(t)-C_{h}(t)\right\|_{\mathcal{L}(\mathcal{H})} \leq \frac{1}{h} \int_{0}^{t}\left\|\widehat{R}_{h}^{(2)}\left(H, G_{h}(., s)\right)\right\|_{\mathcal{L}(\mathcal{H})} d s .
$$

We then deduce that:

$$
\left\|\sigma_{h}^{\text {wick }}\left(B_{h}(t)-C_{h}(t)\right)\right\|_{L^{\infty}\left(\mathbb{R}^{2 n}\right)} \leq\left\|B_{h}(t)-C_{h}(t)\right\|_{\mathcal{L}(\mathcal{H})} \leq M(t) \sqrt{h} I_{h}^{\infty}(A) .
$$

Third step. From Proposition 4.4,

$$
\sigma_{h}^{\text {wick }}\left(\left(C_{h}(t)\right)=e^{\frac{h}{4} \Delta} \sigma_{h}^{\text {weyl }}\left(C_{h}(t)\right)=e^{\frac{h}{4} \Delta} w_{h}(., t) .\right.
$$


Then

$$
\begin{aligned}
\| \sigma_{h}^{\text {wick }}\left(\left(C_{h}(t)\right)-w_{h}(., t) \|_{L^{\infty}\left(\mathbb{R}^{2 n}\right)}\right. & \leq \frac{h}{4} \int_{0}^{1}\left\|\Delta e^{\frac{\theta h}{4} \Delta} w_{h}(., t)\right\|_{L^{\infty}\left(\mathbb{R}^{2 n}\right)} \\
& \leq\left\|\Delta w_{h}(., t)\right\|_{L^{\infty}\left(\mathbb{R}^{2 n}\right)} .
\end{aligned}
$$

In view of the estimates (6.4) satisfied by $w_{h}(., t)$, we obtain:

$$
\left\|\sigma_{h}^{\text {wick }}\left(C_{h}(t)\right)-w_{h}(., t)\right\|_{L^{\infty}\left(\mathbb{R}^{2 n}\right)} \leq M(t) \sqrt{h} I_{h}^{\infty}(A) .
$$

Since $w_{h}(., t)=\left(\sigma_{h}^{\text {wick }}(A)\right) \circ \varphi_{t}$, then inequality (1.12) in Theorem 1.1 arises from (6.3), (6.11) and (6.12).

\section{Proof of Theorem 2.1.}

Let $\rho_{h}(t)$ be a family of solutions to the equation (TDHF) (2.1), satisfying the hypotheses of Theorem 2.1. Let $u_{h}(., t)$ be the function defined in $(2.3)$. Let $v_{h}(., t)$ be the solution to Vlasov equation (2.5) such that $v_{h}(., 0)=u_{h}(., 0)$. We shall use the functions $V_{q}\left(., \rho_{h}(t)\right)$ and $V_{\mathrm{cl}}\left(., v_{h}(., t)\right)$ defined in (2.2) and (2.7), respectively. We shall also use the following functions:

$$
H_{h}^{\mathrm{HF}}(x, \xi, t)=|\xi|^{2}+V_{q}\left(x, \rho_{h}(t)\right), \quad H_{h}^{\mathrm{VL}}(x, \xi, t)=|\xi|^{2}+V_{c l}\left(x, v_{h}(., t)\right),
$$

and the associated operators through the Weyl calculus, namely:

$$
\widehat{H}_{h}^{\mathrm{HF}}=-h^{2} \Delta+V_{q}\left(., \rho_{h}(t)\right), \quad \widehat{H}_{h}^{\mathrm{VL}}=-h^{2} \Delta+V_{c l}\left(., v_{h}(., t)\right),
$$

by using the same notation for the function and the corresponding multiplication operator. We shall denote by $w_{h}(X, t)$ the solution to

$$
\frac{\partial w_{h}}{\partial t}(., t)=\left\{H_{h}^{\mathrm{HF}}(., t), w_{h}(., t)\right\},
$$

such that:

$$
w_{h}(., 0)=v_{h}(., 0)=u_{h}(., 0) .
$$

In order to compare $v_{h}(., t)$ with $w_{h}(., t)$, we note that the Vlasov equation (2.5) is written as:

$$
\frac{\partial v_{h}}{\partial t}(., t)=\left\{H_{h}^{\mathrm{VL}}(., t), v_{h}(., t)\right\} .
$$

We shall use the operator $B_{h}(t)$ solution to

$$
i h \frac{d B_{h}(t)}{d t}=\left[\widehat{H}_{h}^{\mathrm{HF}}(t), B_{h}(t)\right], \quad B_{h}(0)=\mathcal{T}_{h}\left(\rho_{h}(0)\right),
$$

where $\mathcal{T}_{h}$ is the mapping used in Section 5. Finally, we shall also use the following operators:

$$
C_{h}(t)=(2 \pi h)^{n} O p_{h}^{\text {weyl }}\left(w_{h}(., t)\right), \quad D_{h}(t)=(2 \pi h)^{n} O p_{h}^{\text {weyl }}\left(v_{h}(., t)\right) .
$$

According to the point (d) in Theorem 5.1, we have

$$
\sigma_{h}^{\text {weyl }}\left(B_{h}(0)\right)=\sigma_{h}^{\text {weyl }}\left(\mathcal{T}_{h}\left(\rho_{h}(0)\right)\right)=\sigma_{h}^{\text {wick }}\left(\rho_{h}(0)\right)=(2 \pi h)^{n} u_{h}(., 0) .
$$

Consequently,

$$
B_{h}(0)=C_{h}(0)=D_{h}(0)
$$


Theorem 2.1 will be a consequence of the comparison between the Wick symbol of the operators $\rho_{h}(t)$ and $B_{h}(t)$, between those of $B_{h}(t)$ and $C_{h}(t)$, between those of $C_{h}(t)$ and $D_{h}(t)$, and finally between the Wick symbol of $D_{h}(t)$ and the function $v_{h}(., t)$. Each of these comparisons shall be written using the expression $I_{h}^{\operatorname{tr}}\left(\rho_{h}(0)\right)$ defined in (2.8), and shall correspond to one step of the proof, but before that, we need three more lemmas.

Lemma 7.1. Let $\rho_{h}(t)$ be a family of solutions to the (TDHF) equation satisfying the assumptions in Theorem 2.1. Let $v_{h}(., t)$ be the function defined above. Then, for all integer numbers $k \geq 0$ we have:

$$
\left\|\nabla^{k} v_{h}(., t)\right\|_{L^{1}\left(\mathbb{R}^{2 n}\right)} \leq C_{k}(t) h^{-k / 2}\left\|\rho_{h}(0)\right\|_{\mathcal{L}^{1}(\mathcal{H})},
$$

and for all integer numbers $k \geq 1$,

$$
\left\|\nabla^{k} v_{h}(., t)\right\|_{L^{1}\left(\mathbb{R}^{2 n}\right)} \leq C_{k}(t) h^{-(k-1) / 2} I_{h}^{\operatorname{tr}}\left(\rho_{h}(0)\right) .
$$

These estimates remain valid when replacing the function $v_{h}(., t)$ by the function $w_{h}(., t)$.

Proof of the lemma. Since we have (7.4) for $t=0$, then the estimates (7.10) and (7.11) when $t=0$ come from Proposition 4.2 applied with the operator $\rho_{h}(0)$ (for $(7.10)$ ) and with the commutators $\left[P_{j}(h), \rho_{h}(0)\right]$ and $\left[Q_{j}(h), \rho_{h}(0)\right]$ (for $(7.11)$ ). Since the potentials $V$ and $W$ are in $W^{\infty, \infty}\left(\mathbb{R}^{n}\right)$, then the functions $V_{q}\left(., \rho_{h}(t)\right)$ and $V_{\mathrm{cl}}\left(., v_{h}(., t)\right)$ are uniformly bounded together with all of their derivatives. Consequently, the estimates satisfied at $t=0$ by $v_{h}(., 0)=w_{h}(., 0)$ remain valid along the Hamiltonian flows associated to the two symbols $H_{h}^{H F}(., t)$ and $H_{h}^{V L}(., t)$. Thus, the estimates $(7.10)$ and (7.11) remain valid for all $t$ for the function $v_{h}(., t)$ and the function $w_{h}(., t)$.

Lemma 7.2. If $W$ is a function in $W^{\infty, \infty}\left(\mathbb{R}^{n}\right)$, if we denote by $W_{x}$ the multiplication by $y \rightarrow W(x-y)$ and if $A$ is trace class, then

$$
e^{\frac{h}{4} \Delta_{x}} \operatorname{Tr}\left(W_{x} \circ A\right)=(2 \pi h)^{-n} \int_{\mathbb{R}^{2 n}} W(x-y) \sigma_{h}^{\text {wick }}(A)(y, \eta) d y d \eta .
$$

Proof. Let us begin by the case, $A=O p_{h}^{\text {weyl }}(F)$ with $F$ in $W^{\infty, 1}\left(\mathbb{R}^{2 n}\right)$. Then, if $W$ is as in the lemma, by (3.2) we have:

$$
\operatorname{Tr}\left(W_{x} \circ A\right)=(2 \pi h)^{-n} \int_{\mathbb{R}^{2 n}} W(x-y) \sigma_{h}^{\text {weyl }}(A)(y, \eta) d y d \eta,
$$

and then

$$
e^{\frac{h}{4} \Delta_{x}} \operatorname{Tr}\left(W_{x} \circ A\right)=(2 \pi h)^{-n} \int_{\mathbb{R}^{2 n}} W(x-y) e^{\frac{h}{4} \Delta_{x}} \sigma_{h}^{\text {weyl }}(A)(y, \eta) d y d \eta .
$$

Besides,

$$
\int_{\mathbb{R}^{2 n}} W(x-y)\left(e^{\frac{h}{4} \Delta_{\eta}}-I\right) \sigma_{h}^{\text {weyl }}(A)(y, \eta) d y d \eta=0,
$$

and taking into account Proposition 4.4 we deduce (7.12). Suppose now that $A$ is an arbitrarily given trace class operator. Then Theorem 5.2 shows that there exists a sequence of operators $A_{j}$, written as, $A_{j}=O p_{h}^{\text {weyl }}\left(F_{j}\right)$ with $F_{j}$ in $W^{\infty, 1}\left(\mathbb{R}^{2 n}\right)$, converging to $A$ in $\mathcal{L}^{1}(\mathcal{H}),\left(h>0\right.$ being fixed). Equality (7.12) valid for all the $A_{j}$ is also true for $A$ when taking the limit while using Proposition 4.2. 
Lemma 7.3. With the above notations, we have:

$\left\|v_{h}(., t)-w_{h}(., t)\right\|_{L^{1}\left(\mathbb{R}^{2 n}\right)} \leq C(t) I_{h}^{t r}\left(\rho_{h}(0)\right)\left[h+\int_{[0, t]}\left\|v_{h}(., s)-u_{h}(., s)\right\|_{L^{1}\left(\mathbb{R}^{2 n}\right)} d s\right]$.

Proof. We deduce from (7.3) and (7.5) that:

$\frac{\partial\left(v_{h}-w_{h}\right)}{\partial t}(., t)=\left\{H_{h}^{\mathrm{HF}}(., t),\left(v_{h}(., t)-w_{h}(., t)\right)\right\}+\left\{\left(H_{h}^{\mathrm{HF}}(., t)-H_{h}^{\mathrm{VL}}(., t)\right), v_{h}(., t)\right\}$.

From Duhamel principle, since $v_{h}(., 0)-w_{h}(., 0)=0$ and since the Hamiltonian flow associated to the function $H_{h}^{H F}(., t)$ preserves the norm of $L^{1}\left(\mathbb{R}^{2 n}\right)$, we obtain

$$
\left\|v_{h}(., t)-w_{h}(., t)\right\|_{L^{1}\left(\mathbb{R}^{2 n}\right)} \leq \int_{[0, t]}\left\|\left\{H_{h}^{\mathrm{HF}}(., s)-H_{h}^{\mathrm{VL}}(., s), v_{h}(., s)\right\}\right\|_{L^{1}\left(\mathbb{R}^{2 n}\right)} d s .
$$

We have:

$$
\begin{aligned}
H_{h}^{\mathrm{HF}}(., s)-H_{h}^{\mathrm{VL}}(., s)= & \left(I-e^{\frac{h}{4} \Delta_{x}}\right) \operatorname{Tr}\left(W_{x} \circ \rho_{h}(t)\right)+e^{\frac{h}{4} \Delta_{x}} \operatorname{Tr}\left(W_{x} \circ \rho_{h}(t)\right) \\
& -\int_{\mathbb{R}^{2 n}} W(x-y) v_{h}(y, \eta, t) d y d \eta
\end{aligned}
$$

From Lemma 7.2, we have:

$$
e^{\frac{h}{4} \Delta_{x}} \operatorname{Tr}\left(W_{x} \circ \rho_{h}(t)\right)=\int_{\mathbb{R}^{2 n}} W(x-y) u_{h}(y, \eta, t) d y d \eta .
$$

We then deduce:

$$
\begin{aligned}
& H_{h}^{\mathrm{HF}}(x, \xi, s)-H_{h}^{\mathrm{VL}}(x, \xi, s)=\left(I-e^{\frac{h}{4} \Delta_{x}}\right) \operatorname{Tr}\left(W_{x} \circ \rho_{h}(t)\right) \cdots \\
& \quad+\int_{\mathbb{R}^{2 n}} W(x-y)\left(u_{h}(y, \eta, t)-v_{h}(y, \eta, t)\right) d y d \eta .
\end{aligned}
$$

In view of the preceding points,

$$
\begin{aligned}
& \left\|v_{h}(., t)-w_{h}(., t)\right\|_{L^{1}\left(\mathbb{R}^{2 n}\right)} \\
& \left.\quad \leq C \int_{[0, t]} \| \nabla v_{h}(., s)\right\} \|_{L^{1}\left(\mathbb{R}^{2 n}\right)}\left[h+\left\|u_{h}(., s)-v_{h}(., s)\right\|_{L^{1}\left(\mathbb{R}^{2 n}\right)}\right] d s .
\end{aligned}
$$

From Lemma 7.1 (with $k=1$ ) we then deduce (7.13).

End of the proof of Theorem 2.1. First step. With the above notations (TDHF) equation is written as:

$$
i h \frac{d \rho_{h}(t)}{d t}=\left[\widehat{H}_{h}^{\mathrm{HF}}(t), \rho_{h}(t)\right]
$$

We consequently have:

$$
i h \frac{d\left(\rho_{h}(t)-B_{h}(t)\right)}{d t}=\left[\widehat{H}_{h}^{\mathrm{HF}}(t),\left(\rho_{h}(t)-B_{h}(t)\right)\right] .
$$

Since the propagator associated to this equation preserves the trace norm, and since $B_{h}(0)=\mathcal{T}_{h}\left(\rho_{h}(0)\right)$, we then deduce that:

$$
\left\|\rho_{h}(t)-B_{h}(t)\right\|_{\mathcal{L}^{1}(\mathcal{H})} \leq\left\|\rho_{h}(0)-\mathcal{T}_{h}\left(\rho_{h}(0)\right)\right\|_{\mathcal{L}^{1}(\mathcal{H})} .
$$


Consequently, from the Proposition 4.2 (with $\alpha=0$ ) and Theorem 5.1 (point c)), (7.14)

$(2 \pi h)^{-n}\left\|\sigma_{h}^{\text {wick }}\left(\rho_{h}(t)-B_{h}(t)\right)\right\|_{L^{1}\left(\mathbb{R}^{2 n}\right)} \leq\left\|\rho_{h}(0)-\mathcal{T}_{h}\left(\rho_{h}(0)\right)\right\|_{\mathcal{L}^{1}(\mathcal{H})} \leq C \sqrt{h} I_{h}^{\operatorname{tr}}\left(\rho_{h}(0)\right)$.

Second step. We shall bound in norm $B_{h}(t)-C_{h}(t)$, and towards this aim, we shall show that $B_{h}(t)$ and $C_{h}(t)$ verify similar equations. The operator $B_{h}(t)$ verifies (7.6) whereas:

$$
i h \frac{d C_{h}(t)}{d t}=i h(2 \pi h)^{n} O P_{h}^{\mathrm{weyl}}\left(\left\{H_{h}^{\mathrm{HF}}(., t), w_{h}(., t)\right\}\right) .
$$

With the notation (6.6) we have:

$$
\begin{aligned}
& \left.O P_{h}^{\text {weyl }}\left(H_{h}^{\mathrm{HF}}(., t)\right), O P_{h}^{\text {weyl }}\left(w_{h}(., t)\right)\right] \\
& \quad=\frac{h}{i} O P_{h}^{\mathrm{weyl}}\left(\left\{H_{h}^{\mathrm{HF}}(., t), w_{h}(., t)\right\}\right)+\widehat{R}_{h}^{(2)}\left(H_{h}^{\mathrm{HF}}(., t), w_{h}(., t)\right) .
\end{aligned}
$$

Consequently,

$$
i h \frac{d C_{h}(t)}{d t}-\left[\widehat{H}_{h}^{\mathrm{HF}}(t), C_{h}(t)\right]=(2 \pi h)^{n} \widehat{R}_{h}^{(2)}\left(H_{h}^{\mathrm{HF}}(., t), w_{h}(., t)\right) .
$$

We know that $R_{h}^{(2)}(F, G)=0$ for all functions $G$ when $F(x, \xi)=|\xi|^{2}$. We can then replace $H_{h}^{\mathrm{HF}}(., t)$ by $V_{q}\left(., \rho_{h}(t)\right)$ in the right-hand side of (7.15). By combining (7.15) with equation (7.6) verified by $B_{h}(t)$ and using Duhamel principle and (7.4), we obtain

$$
\left\|B_{h}(t)-C_{h}(t)\right\|_{\mathcal{L}^{1}(\mathcal{H})} \leq \frac{1}{h}(2 \pi h)^{n} \int_{[0, t]}\left\|\widehat{R}_{h}^{(2)}\left(V_{q}\left(., \rho_{h}(s)\right), w_{h}(., s)\right)\right\|_{\mathcal{L}^{1}(\mathcal{H})} d s .
$$

From Theorem 3.1 of [1] applied with $N=2, F=V_{q}\left(., \rho_{h}(s)\right), G=w_{h}(., s), p=\infty$, $q=1$, we have

$$
\begin{aligned}
& \left\|\widehat{R}_{h}^{(2)}\left(V_{q}\left(., \rho_{h}(s)\right), w_{h}(., s)\right)\right\|_{\mathcal{L}^{1}(\mathcal{H})} \\
& \quad \leq C h^{-n} \sum_{\substack{\alpha+\beta \leq 6 n+10 \\
\alpha \geq 3, \beta \geq 3}} h^{(\alpha+\beta) / 2}\left\|\nabla^{\alpha} V_{q}\left(., \rho_{h}(s)\right)\right\|_{L^{\infty}\left(\mathbb{R}^{n}\right)}\left\|\nabla^{\beta} w_{h}(., s)\right\|_{L^{1}\left(\mathbb{R}^{2 n}\right)} .
\end{aligned}
$$

Since the potentials $V$ and $W$ are in $W^{\infty, \infty}\left(\mathbb{R}^{n}\right)$ and since the $L^{1}\left(\mathbb{R}^{2 n}\right)$ norm of $w_{h}(., s)$ is bounded (Lemma 7.1) then the derivatives of all order of $V_{q}\left(., \rho_{h}(s)\right)$ are bounded. For $\beta \geq 1$, the function $\nabla^{\beta} w_{h}(., s)$ verifies the estimates (7.11) of Lemma 7.1. Consequently, when $h \in(0,1]$,

$$
\left\|\widehat{R}_{h}^{(2)}\left(V_{q}\left(., \rho_{h}(s)\right), w_{h}(., s)\right)\right\|_{\mathcal{L}^{1}(\mathcal{H})} \leq C(s) h^{3 / 2} I_{h}^{t r}\left(\rho_{h}(0)\right) .
$$

Consequently,

$$
\left\|B_{h}(t)-C_{h}(t)\right\|_{\mathcal{L}^{1}(\mathcal{H})} \leq C(t) \sqrt{h} I_{h}^{t r}\left(\rho_{h}(0)\right) .
$$

We then deduce (from the Proposition 4.2 with $\alpha=0$ ) that:

$$
(2 \pi h)^{-n}\left\|\sigma_{h}^{\text {wick }}\left(B_{h}(t)-C_{h}(t)\right)\right\|_{L^{1}\left(\mathbb{R}^{2 n}\right)} \leq C(t) \sqrt{h} I_{h}^{t r}\left(\rho_{h}(0)\right) .
$$

Third step. From the Proposition 4.4, we have

$$
\begin{aligned}
& (2 \pi h)^{-n}\left\|\sigma_{h}^{\text {wick }}\left(C_{h}(t)-D_{h}(t)\right)\right\|_{L^{1}\left(\mathbb{R}^{2 n}\right)} \\
& \quad \leq \| e^{\frac{h}{4} \Delta}\left(v_{h}(., t)-w_{h}(., t)\left\|_{L^{1}\left(\mathbb{R}^{2 n}\right)} \leq\right\|\left(v_{h}(., t)-w_{h}(., t) \|_{L^{1}\left(\mathbb{R}^{2 n}\right)} .\right.\right.
\end{aligned}
$$


Then, from Lemma 7.2,

$$
\begin{aligned}
& (2 \pi h)^{-n}\left\|\sigma_{h}^{\text {wick }}\left(C_{h}(t)-D_{h}(t)\right)\right\|_{L^{1}\left(\mathbb{R}^{2 n}\right)} \\
& \quad \leq C I_{h}\left(\rho_{h}(0)\right)\left[h+\int_{[0, t]}\left\|u_{h}(., s)-v_{h}(., s)\right\|_{L^{1}\left(\mathbb{R}^{2 n}\right)} d s\right] .
\end{aligned}
$$

Fourth step. From Proposition 4.4, we have

$$
(2 \pi h)^{-n} \sigma_{h}^{\text {wick }}\left(D_{h}(t)\right)=(2 \pi h)^{-n} e^{\frac{h}{4} \Delta} \sigma_{h}^{\text {weyl }}\left(D_{h}(t)\right)=e^{\frac{h}{4} \Delta} v_{h}(., t) .
$$

Consequently, from the Lemma 7.1 with $k=2$, we obtain

$$
\begin{aligned}
& \| v_{h}(., t)-(2 \pi h)^{-n} \sigma_{h}^{\text {wick }}\left(D_{h}(t)\left\|_{L^{1}\left(\mathbb{R}^{2 n}\right)} \leq\right\|\left(e^{\frac{h}{4} \Delta}-I\right) v_{h}(., t) \|_{L^{1}\left(\mathbb{R}^{2 n}\right)} \leq \ldots\right. \\
& \quad \leq C h\left\|\nabla^{2} v_{h}(., t)\right\|_{L^{1}\left(\mathbb{R}^{2 n}\right)} \leq C(t) \sqrt{h} I_{h}^{\operatorname{tr}}\left(\rho_{h}(0)\right) .
\end{aligned}
$$

Since $u_{h}(., t)$ is defined by (2.3), we get from estimates (7.14), (7.16), (7.17) and (7.18) obtained in the four steps that:

$$
\begin{aligned}
& \left\|u_{h}(., t)-v_{h}(., t)\right\|_{L^{1}\left(\mathbb{R}^{2 n}\right)} \\
& \quad \leq C(t) I_{h}^{t r}\left(\rho_{h}(0)\right)\left[\sqrt{h}+\int_{[0, t]}\left\|u_{h}(., s)-v_{h}(., s)\right\|_{L^{1}\left(\mathbb{R}^{2 n}\right)} d s\right] .
\end{aligned}
$$

From Gronwall lemma, we have

$$
\left\|u_{h}(., t)-v_{h}(., t)\right\|_{L^{1}\left(\mathbb{R}^{2 n}\right)} \leq C(t) \sqrt{h} I_{h}^{t r}\left(\rho_{h}(0)\right) e^{C(t) I_{h}\left(\rho_{h}(0)\right)},
$$

when $h \in(0,1]$, with a different constant $C(t)$. Theorem 2.1 is complete.

\section{Acknowledgment}

The authors would like to express their thanks to the referee for useful remarks.

\section{References}

[1] L. Amour, M. Khodja and J. Nourrigat, The semiclassical limit of the time dependent HartreeFock equation. The Weyl symbol of the solution, Anal. Partial Differ. Equ. (to be published).

[2] R. Beals, Characterization of pseudodifferential operators and applications, Duke Math. J. 44(1) (1977), 45-57.

[3] F.A. Berezin, Quantization, Izv. Akad. Nauk SSSR Ser. Mat., 38 (1974), 1116-1175.

[4] A. Bouzouina and D. Robert, Uniform semiclassical estimates for the propagation of quantum observables, Duke Math. J. 111(2) (2002), 223-252.

[5] A. Bove, G. Da Prato, and G. Fano, On the Hartree-Fock time-dependent problem, Comm. Math. Phys. 49(1) (1976), 25-33.

[6] A.-P. Calderón and R. Vaillancourt, A class of bounded pseudo-differential operators, Proc. Natl. Acad. Sci. USA, 69 (1972), 1185-1187.

[7] C. Cohen-Tannoudji, B. Diu and F. Laloë, Mécanique quantique, Hermann, Paris, 1973.

[8] M. Combescure and D. Robert, Quadratic quantum Hamiltonians revisited, Cubo 8(1) (2006), 61-86.

[9] A. Córdoba and C. Fefferman, Wave packets and Fourier integral operators, Commun. Partial Differ. Equ. 3(11) (1978), 979-1005.

[10] G.B. Folland, Harmonic analysis in phase space, Annals of Mathematics Studies, 122, Princeton University Press, Princeton, NJ, 1989.

[11] L. Hörmander, The analysis of linear partial differential operators, IV, Grundlehren der Mathematischen Wissenschaften 275 [Fundamental Principles of Mathematical Sciences], Springer-Verlag, Berlin (1985), ISBN 3-540-13829-3, Fourier integral operators. 
[12] N. Lerner, Some facts about the Wick calculus, in Pseudo-differential operators, 135-174, Lecture Notes in Math., 1949 , Springer, Berlin, 2008.

[13] D. Robert, Autour de l'approximation semi-classique, Progress in Mathematics, 68, Birkhäuser Boston Inc., Boston, MA, 1987.

[14] C. Rondeaux, Classes de Schatten d'opérateurs pseudo-différentiels, Ann. Sci. École Norm. Sup. (4) $\mathbf{1 7}(1)$ (1984), 67-81.

[15] M.A. Shubin, Pseudodifferential operators and spectral theory, Springer Series in Soviet Mathematics, Springer-Verlag, Berlin, 1987, Translated from the Russian by Stig I. Andersson.

[16] A. Unterberger, Les opérateurs métadifférentiels, in 'Complex Analysis, Microlocal Calculus and Relativistic Quantum Theory' (Proc. Internat. Colloq., Centre Phys., Les Houches, 1979), Lecture Notes in Physics, 126, 205-241, Springer, Berlin, 1980.

[17] M. Zworski, Semiclassical analysis, Graduate Studies in Mathematics, 138, American Mathematical Society, Providence, RI, 2012.

LMR EA 4535 AND FR-CNRS 3399

Université de Reims Moulin de la Housse

BP 1039, 51687 REIMS, CEDEX 2

FRANCE

E-mail address: laurent.amour@univ-reims.fr

E-mail address: mohamed.khodja@univ-reims.fr

E-mail address: jean.nourrigat@univ-reims.fr 
